PROCEEDINGS OF THE

AMERICAN MATHEMATICAL SOCIETY

Volume 129, Number 2, Pages 483-493

S 0002-9939(00)05545-3

Article electronically published on August 29, 2000

\title{
SOLUTION OF A FUNCTIONAL EQUATION ARISING IN AN AXIOMATIZATION OF THE UTILITY OF BINARY GAMBLES
}

\author{
JÁNOS ACZÉL, GYULA MAKSA, AND ZSOLT PÁLES
}

(Communicated by David R. Larson)

\begin{abstract}
For a new axiomatization, with fewer and weaker assumptions, of binary rank-dependent expected utility of gambles the solution of the functional equation

$$
(z / p) \gamma^{-1}[z \gamma(p)]=\varphi^{-1}[\varphi(z) \psi(p)] \quad(z, p \in] 0,1[)
$$

is needed under some monotonicity and surjectivity conditions. We furnish the general such solution and also the solutions under weaker suppositions. In the course of the solution we also determine all sign preserving solutions of the related general equation
\end{abstract}

$$
h(u)[g(u+v)-g(v)]=f(v) g(u+v) \quad\left(u \in \mathbb{R}_{+}, v \in \mathbb{R}\right) .
$$

\section{INTRODUCTION}

Marley and Luce 4] considered the following problem concerning preferences among binary gambles. Let $\mathcal{C}$ denote the set of consequences, and let $\mathcal{E}_{E}$ be an algebra of subsets of $E$, the universal set of a chance experiment. Let $(x, C ; y)$ denote a binary, uncertain alternative, also called a binary gamble, in which $x \in \mathcal{C}$ is the consequence to the decision maker if event $C \in \mathcal{E}_{E}$ occurs and $y$ is the consequence if $\bar{C}=E \backslash C$ occurs. They also considered compound gambles of the form $(\xi, D ; \eta)$, where $\xi=(x, C ; y), \eta=\left(x^{\prime}, C^{\prime} ; y^{\prime}\right)$ and the events $D, C$, and $C^{\prime}$ arise from independent realizations of the underlying chance experiment. Let $e \in \mathcal{C}$ denote a distinguished element called the status quo. Let $\succsim$ be a preference order over the set $\mathcal{G}$ of all such first- and second-order binary gambles and consequences from $\mathcal{C}$, where $x \succsim e$ for all $x \in \mathcal{C}$. Thus, all the consequences under consideration, and so gambles, are perceived as gains. Let $\mathcal{G}^{*}$ denote the subset of gambles with $y=e$.

Received by the editors October 23, 1998 and, in revised form, April 27, 1999.

2000 Mathematics Subject Classification. Primary 39B22, 39B72, 39B12; Secondary 26A51, $91 \mathrm{~B} 16$.

Key words and phrases. Functional equation, binary gamble, convexity.

This research has been supported in part by the Natural Sciences and Engineering Research Council (NSERC) of Canada Grant OGP 002972, by the Hungarian National Research Science Foundation (OTKA) Grant T-016846 and by the Fund for Development and Research in Higher Education (FKFP) Grant 0310/1997. The authors are grateful to R. Duncan Luce (University of California, Irvine) for the problem and for advice, in particular regarding the Introduction. 
They assume sufficient structure on the restriction of $\succsim$ to $\mathcal{G}^{*}$ so that over $\mathcal{G}^{*}$ there is a "separable", order-preserving representation $u w$ with surjective $u: \mathcal{G}^{*} \longrightarrow I$, where $I$ is dense in a real interval $[0, d[, u(e)=0$, and surjective $w: \mathcal{E}_{E} \longrightarrow J$, where $J$ is a dense subset of $[0,1]$ that includes 0 and 1 . The conditions needed are well known (see e.g. [4]) and we do not go into them here. They were able, using elementary axioms, to extend these maps (and the preservation of order) onto $[0, d[$ and $[0,1]$, respectively. With that in hand, they attacked the problem of finding conditions so that this representation $u w$ can be extended to $\mathcal{G}$ as

$$
U(\xi, C ; \eta)=U(\xi) W(E)+U(\eta)[1-W(C)], \quad \xi \succsim \eta,
$$

where, for some $k>0, U=u^{k}$ and $W=w^{k}$.

They impose two additional, necessary conditions. The first, called gains partition, asserts the following. There exists a bijection $M$ of $\mathcal{E}_{E}$ onto itself that inverts the order $\succsim_{\mathcal{E}}$ (induced on $\mathcal{E}_{E}$ by $\succsim$ ) such that, for first-order gambles $\xi, \xi^{\prime}, \eta, \eta^{\prime}$ with $\xi \succsim \eta, \xi^{\prime} \succsim \eta^{\prime}$ and for events $C, C^{\prime} \in \mathcal{E}_{E}$ if

$$
(\xi, C ; e) \sim\left(\xi^{\prime}, C^{\prime} ; e\right) \quad \text { and } \quad(\eta, M(C) ; e) \sim\left(\eta^{\prime}, M\left(C^{\prime}\right) ; e\right),
$$

then

$$
(\xi, C ; \eta) \sim\left(\xi^{\prime}, C ; \eta^{\prime}\right)
$$

The second, called event commutativity, is

$$
((x, C ; y), D ; y) \sim((x, D ; y), C ; y) .
$$

Their proof that separability plus (21) and (31) are sufficient to yield (11) entails the following major steps: Hypothesis (2) is recast as a statement on a numerical function $R$ such that, for first-order gambles $\xi \succsim \eta$,

$$
U(\xi, C ; \eta)=R[U(\xi) W(C), U(\eta) W(M(C))] .
$$

Define

$$
\begin{aligned}
p & =W(C), \quad z=\frac{U(\eta)}{U(\xi)} \leq 1, \\
\pi(p) & \left.\left.=W\left[\overline{W^{-1}(p)}\right]=W(M(C)), \quad \gamma(p)=\frac{\pi(p)}{p}, \quad \gamma:\right] 0,1\right] \rightarrow[0, \infty[, \\
H(z, p) & \left.\left.=\frac{z}{p} \gamma^{-1}[z \gamma(p)] \quad(z \in[0,1], p \in] 0,1\right]\right) .
\end{aligned}
$$

Using (3) and (4), Marley and Luce show in [4 also that $H$ (there $G$ ) satisfies the usual permutability functional equation (see [1, pp. 270-273]) and so for some strictly increasing $\varphi:] 0,1\left[\rightarrow \mathbb{R}_{+}\left(\mathbb{R}_{+}\right.\right.$is the set of positive reals) and strictly decreasing $\psi:] 0,1[\rightarrow] 1, \infty\left[, H(z, p)=\varphi^{-1}[\varphi(z) \psi(p)]\right.$. In view of this, we exclude $z=0, z=1$, and $p=1$ in what follows. This does no harm, since equation (5 below is satisfied also for $z=0, z=1$ or $p=1$ if $\gamma(1)=0, \varphi(0)=0, \varphi(1)=\infty$, and $\psi(1)=1$, which conforms with our final result (see Section 5). Marley and Luce (4]) prove also that $\gamma:] 0,1\left[\rightarrow \mathbb{R}_{+}\right.$is onto (surjective), strictly decreasing, and that

$$
\lim _{p \rightarrow 0}[p \gamma(p)]=\lim _{p \rightarrow 0} \pi(p)=1, \quad \lim _{z \rightarrow 1} \varphi(z)=\infty .
$$


Thus, they were interested in solutions with the above properties of the following functional equation in three unknown functions:

$$
\frac{z}{p} \gamma^{-1}[z \gamma(p)]=\varphi^{-1}[\varphi(z) \psi(p)] \quad(z, p \in] 0,1[) .
$$

Finding that solution is the purpose of the present paper. Marley and Luce show from our solution that their desired result follows.

\section{Reduction to a "Linear" equation}

We prefer to write equation (5) in the form

$$
\varphi\left((z / p) \gamma^{-1}[z \gamma(p)]\right)=\varphi(z) \psi(p) \quad(z, p \in] 0,1[)
$$

because it shows that all values of $\varphi(z) \psi(p)$ are also values of the function $\varphi$ (that is, $\varphi(z) \psi(p)$ is in the codomain of $\varphi$ ). Since we had supposed and will continue to suppose that $\varphi$ is strictly monotonic, we can indeed take its inverse and write (5') as (5).

On the other hand, we will not presuppose either the direction of monotonicity (increasing or decreasing) of $\varphi$ and $\psi$ or the exact codomains of $\varphi$ and $\psi$ (we will only suppose that they map $] 0,1\left[\right.$ into $\mathbb{R}_{+}$). These will turn out to be consequences of (5-1) and of the other conditions.

Proposition 1. Let the functions $\varphi, \psi:] 0,1\left[\rightarrow \mathbb{R}_{+}\right.$be strictly monotonic, let $\gamma:$ ] $0,1\left[\rightarrow \mathbb{R}_{+}\right.$be surjective and strictly decreasing and let (5) be satisfied. Then and only then the functions $F, G: \mathbb{R} \rightarrow \mathbb{R}$ and $\Phi: \mathbb{R}_{+} \rightarrow \mathbb{R}$ defined by

$$
\begin{aligned}
& \Phi(u)=\ln \varphi\left(e^{-u}\right) \quad\left(u \in \mathbb{R}_{+}\right), \\
& F(v)=\ln \psi\left(\gamma^{-1}\left(e^{-v}\right)\right) \quad(v \in \mathbb{R}),
\end{aligned}
$$

and

$$
G(v)=v-\ln \gamma^{-1}\left(e^{-v}\right) \quad(v \in \mathbb{R})
$$

satisfy

$$
\Phi(u)+F(v)=\Phi(G(u+v)-G(v)) \quad\left(u \in \mathbb{R}_{+}, v \in \mathbb{R}\right) ;
$$

furthermore $\Phi, F$ are strictly monotonic and $G$ is strictly increasing.

Proof. The strict monotonicity of $\Phi$ and $F$ is obvious. Substituting (6), (77) and (8) into (5) with $z=e^{-u}, p=\gamma^{-1}\left(e^{-v}\right)$, we get

$$
\left.\varphi^{-1}\left(e^{\Phi(u)+F(v)}\right)=e^{-(G(u+v)-G(v))} \in\right] 0,1[.
$$

Therefore $G$ is indeed strictly increasing and, applying $\varphi$ and then $\ln$ to both sides of (10), we get (19). 


\section{Differentiability AND A DIFFERENTIAL-FUNCTIONAL EQUATION}

Proposition 2. Let $F: \mathbb{R} \rightarrow \mathbb{R}, \Phi: \mathbb{R}_{+} \rightarrow \mathbb{R}$ be strictly monotonic, $G: \mathbb{R} \rightarrow$ $\mathbb{R}$ strictly increasing solutions of (9). Then $\Phi, G$ and $F$ are differentiable and $G$ is either everywhere strictly convex on $\mathbb{R}$ or everywhere strictly concave on $\mathbb{R}$. Furthermore

and

$$
\Phi^{\prime}(u)\left[G^{\prime}(u+v)-G^{\prime}(v)\right]=F^{\prime}(v) G^{\prime}(u+v) \quad\left(u \in \mathbb{R}_{+}, v \in \mathbb{R}\right) .
$$

We prove this proposition through several steps:

Step 1. The function $G$ is either strictly convex or strictly concave everywhere.

Proof. Indeed, if $\Phi$ and $F$ are both strictly increasing, then (9) implies, for all $u \in \mathbb{R}_{+}, v \in \mathbb{R}$, that

$$
\begin{aligned}
\Phi(G(u+(u+v))-G(u+v)) & =\Phi(u)+F(u+v) \\
& >\Phi(u)+F(v)=\Phi(G(u+v)-G(v)),
\end{aligned}
$$

that is,

$$
G(2 u+v)+G(v)>2 G(u+v) \quad\left(u \in \mathbb{R}_{+}, v \in \mathbb{R}\right) .
$$

Thus $G$ is strictly Jensen convex. The same is true if $\Phi$ and $F$ are both strictly decreasing, while the inequality in (15) reverses if one of $\Phi, F$ strictly increases, the other strictly decreases: then $G$ is strictly Jensen concave.

Since $G$ is monotonic, thus locally bounded, $G$ is everywhere strictly convex or concave, respectively. (See Roberts-Varberg [6, p. 219] and Kuczma [3, p. 145].)

Thus (see also [6] pp. 4-7] and [3, p. 156])

(i) $G$ has left and right derivatives at every point,

(ii) $G$ is differentiable at all but at most countably many points,

and, since $G$ is both strictly increasing and convex (concave),

(iii) $G_{+}^{\prime}(v)>0$ for all $v \in \mathbb{R}$.

Step 2. The function $\Phi$ is everywhere differentiable on its domain.

Proof. For fixed $u \in \mathbb{R}_{+}$the function

$$
v \mapsto G(u+v)-G(v)
$$

is positive (because $G$ is strictly increasing) continuous and strictly monotonic. The last two are consequences of the strict convexity or concavity of $G$. For continuity, see [6] pp. 4-7]. As to the strict monotonicity of (16), let $G$ be, say, strictly concave. Then the difference quotients with constant span strictly decrease:

$$
\frac{G(v+u)-G(v)}{u}>\frac{G\left(v^{\prime}+u\right)-G\left(v^{\prime}\right)}{u} \quad\left(v<v^{\prime} \in \mathbb{R}, u \in \mathbb{R}_{+}\right),
$$

i.e., (16) indeed strictly decreases. Therefore the codomain of the function $v \mapsto$ $G(u+v)-G(v)(v \in \mathbb{R})$ is an interval of positive length. 
Since $\Phi$ is monotonic, by Lebesgue's theorem ([5, pp. 5-9], [2, p. 264]) it is differentiable at almost every point of this interval. On the other hand (cf. (ii)), $G$ is differentiable everywhere with the possible exception of countably many points. So for every $u \in \mathbb{R}_{+}$there exists a $v \in \mathbb{R}$ such that $G$ is differentiable at $u+v$ and $\Phi$ is differentiable at $G(u+v)-G(v)$. Thus, by (9) and by the chain rule of differentiation, $\Phi$ is indeed differentiable at every $u \in \mathbb{R}_{+}$.

Step 3. The function $G$ is everywhere differentiable with positive derivative; $\Phi^{\prime}$ is nowhere zero.

Proof. Differentiate (9) with respect to $u$ from the right or left to obtain (remember, by Step 2, $\Phi$ is already differentiable everywhere)

$$
\begin{array}{ll}
\Phi^{\prime}(u)=\Phi^{\prime}(G(u+v)-G(v)) G_{+}^{\prime}(u+v) & \left(u \in \mathbb{R}_{+}, v \in \mathbb{R}\right), \\
\Phi^{\prime}(u)=\Phi^{\prime}(G(u+v)-G(v)) G_{-}^{\prime}(u+v) & \left(u \in \mathbb{R}_{+}, v \in \mathbb{R}\right),
\end{array}
$$

respectively. This will give the desired $G_{+}^{\prime}=G_{-}^{\prime}$; we have only to exclude that $\Phi^{\prime}$ could be somewhere 0 . Suppose, on the contrary, that there exists a $u_{0}>0$ with $\Phi^{\prime}\left(u_{0}\right)=0$. Then, by (iii) and (17) $\Phi^{\prime}$ would be zero on the proper interval $\left\{G\left(u_{0}+v\right)-G(v) \mid v \in \mathbb{R}\right\}$, so $\Phi$ could not be strictly monotonic, contradicting the supposition. Thus (11) holds ( $\Phi^{\prime}$ is nowhere zero) and $G_{+}^{\prime}=G_{-}^{\prime}$, i.e., $G$ is differentiable everywhere on $\mathbb{R}$. Furthermore, from (iii) we have $G^{\prime}>0$ everywhere, that is, 12 holds.

Step 4. The function $F$ is everywhere differentiable with nonzero derivative and (14) holds.

Proof. By Step 3, (17) becomes

$$
\Phi^{\prime}(u)=\Phi^{\prime}(G(u+v)-G(v)) G^{\prime}(u+v) \quad\left(u \in \mathbb{R}_{+}, v \in \mathbb{R}\right) .
$$

On the other hand, (9) and the differentiability of $\Phi$ and $G$ imply also that of $F$. So, differentiating (9) with respect to $v$ we get

$$
F^{\prime}(v)=\Phi^{\prime}(G(u+v)-G(v))\left(G^{\prime}(u+v)-G^{\prime}(v)\right) \quad\left(u \in \mathbb{R}_{+}, v \in \mathbb{R}\right) .
$$

Multiplying (18) by $G^{\prime}(u+v)-G^{\prime}(v)$ and substracting (19) multiplied by $G^{\prime}(u+v)$, we have proved (14). Finally, suppose for contradiction that there exists a $v_{0} \in \mathbb{R}$ with $F^{\prime}\left(v_{0}\right)=0$. Then, by (11) and (14), $G^{\prime}\left(u+v_{0}\right)=G^{\prime}\left(v_{0}\right)$ would hold for all $u \in \mathbb{R}_{+}$, i.e., $G^{\prime}$ would be constant on $\left[v_{0},+\infty[\right.$; thus, by (14) and (12), we would have $F^{\prime}=0$ on $\left[v_{0},+\infty[\right.$ : contradiction, since $F$ is strictly monotonic. This proves (13) and concludes the proof of Proposition 2.

\section{A general Functional EQUATion AND its SOlution}

As a generalization of (14), we consider now the functional equation

$$
h(u)[g(u+v)-g(v)]=f(v) g(u+v) \quad\left(u \in \mathbb{R}_{+}, v \in \mathbb{R}\right) .
$$

In view of (11), (12), (13), we look for solutions without zeros and thus not changing sign. We use the notation $\left.\mathbb{R}_{-}:=\right]-\infty, 0[$. 
Theorem 3. Let the functions $f$ and $g$ be defined on $\mathbb{R}, h$ on $\mathbb{R}_{+}$, each taking values (independently) only in $\mathbb{R}_{+}$or only in $\mathbb{R}_{-}$. Then the general solution of (20) is given by

$$
\begin{array}{ll}
f(v)=\frac{a e^{\lambda v}}{\mu+e^{\lambda v}} & (v \in \mathbb{R}), \\
g(v)=\frac{b}{\mu+e^{\lambda v}} \quad(v \in \mathbb{R}),
\end{array}
$$

and

$$
h(u)=\frac{a}{1-e^{\lambda u}} \quad\left(u \in \mathbb{R}_{+}\right),
$$

where $\mu$ is an arbitrary nonnegative constant while $a, b, \lambda$ are arbitrary nonzero constants.

Proof. Writing (20) in the form

$$
g(u+v)[h(u)-f(v)]=h(u) g(v) \quad\left(u \in \mathbb{R}_{+}, v \in \mathbb{R}\right),
$$

we see that $h\left(u_{0}\right)=f\left(v_{0}\right)$ would imply $h\left(u_{0}\right)=0$ or $g\left(v_{0}\right)=0$ but, by supposition, $g$ and $h$ have no zeros. So

$$
h(u) \neq f(v) \quad\left(u \in \mathbb{R}_{+}, v \in \mathbb{R}\right)
$$

and (24) can be written as

$$
g(u+v)=\frac{h(u) g(v)}{h(u)-f(v)} \quad\left(u \in \mathbb{R}_{+}, v \in \mathbb{R}\right) .
$$

In particular, for $v=0$,

$$
g(u)=\frac{g(0) h(u)}{h(u)-f(0)}=\frac{g(0)}{1-f(0) / h(u)} \quad\left(u \in \mathbb{R}_{+}\right)
$$

and so (25) becomes, by dividing both sides by $g(0) h(u) h(v)$ and taking reciprocals,

$$
h(u) h(v) \frac{h(u+v)-f(0)}{h(u+v)}=(h(u)-f(v))(h(v)-f(0)) \quad\left(u \in \mathbb{R}_{+}, v \in \mathbb{R}_{+}\right) .
$$

We look now for a functional equation for $h$ alone. We use the symmetry of the left-hand side of (27) for this purpose. The symmetry of the left-hand side then gives

$$
\begin{aligned}
& h(u) h(v)-f(0) h(u)-f(v)(h(v)-f(0)) \\
& \quad=h(u) h(v)-f(0) h(v)-f(u)(h(u)-f(0)),
\end{aligned}
$$

that is, (since $f(0) \neq 0$ )

$$
h(v)-f(v) \frac{h(v)-f(0)}{f(0)}=h(u)-f(u) \frac{h(u)-f(0)}{f(0)}=a \quad\left(u \in \mathbb{R}_{+}, v \in \mathbb{R}_{+}\right)
$$

(constant because one side contains only $v$, the other only $u$ ). This gives

$$
f(v)=f(0) \frac{h(v)-a}{h(v)-f(0)} \quad\left(v \in \mathbb{R}_{+}\right) .
$$

Putting this into (27) we get

$$
1-\frac{f(0)}{h(u+v)}=\frac{h(u)(h(v)-f(0))-f(0)(h(v)-a)}{h(u) h(v)}=1-\frac{f(0)}{h(v)}-f(0) \frac{h(v)-a}{h(u) h(v)} .
$$


This is our functional equation for $h$. We simplify it by introducing

$$
\ell(u)=\frac{1}{h(u)} \quad\left(u \in \mathbb{R}_{+}\right) .
$$

Using $f(0) \neq 0$, we obtain the functional equation (see e.g. Aczél [1, pp. 59-61])

$$
\ell(u+v)=\ell(u)+\ell(v)-a \ell(u) \ell(v) \quad\left(u \in \mathbb{R}_{+}, v \in \mathbb{R}_{+}\right) .
$$

By the supposition about $h$, also

$$
\ell(u) \in \mathbb{R}_{+} \quad \text { for all } u \in \mathbb{R}_{+} \quad \text { or } \quad \ell(u) \in \mathbb{R}_{-} \quad \text { for all } u \in \mathbb{R}_{+} .
$$

We show now that $a=0$ is impossible, that is,

$$
a \neq 0 .
$$

Indeed, if $a=0$, then (30) would reduce to the Cauchy equation with $\ell(u)=\kappa u$ $(\kappa \neq 0$ is a constant) as the only solution satisfying (31). This would give, by (29) and (26),

$$
h(u)=\frac{1}{\kappa u} \quad\left(u \in \mathbb{R}_{+}\right), \quad g(v)=\frac{g(0)}{1-f(0) \kappa v} \quad\left(v \in \mathbb{R}_{+}\right) .
$$

Although we established the second equation only for $v \in \mathbb{R}_{+}$, it would follow for all $v \in \mathbb{R}$, so: For an arbitrary $v \in \mathbb{R}$ take a $u \in \mathbb{R}_{+}$such that $u+v \in \mathbb{R}_{+}$. Then from (24) and (33)

$$
g(0)\left(\frac{1}{\kappa u}-f(v)\right)=\frac{1-f(0) \kappa(u+v)}{\kappa u} g(v)=\left(\frac{1}{\kappa u}-f(0)\left(1+\frac{v}{u}\right)\right) g(v) .
$$

Letting $u \rightarrow+\infty$, we would get $-g(0) f(v)=-f(0) g(v)$, that is,

$$
f(v)=\frac{f(0)}{g(0)} g(v) \quad(v \in \mathbb{R}) .
$$

Putting this into (34) would give

$$
g(v)(1-f(0) \kappa(u+v)+f(0) \kappa u)=g(0) \quad\left(u \in \mathbb{R}_{+}, v \in \mathbb{R}\right),
$$

that is, the second equation of (33) for all $v \in \mathbb{R}$ :

$$
g(v)=\frac{g(0)}{1-f(0) \kappa v} \quad(v \in \mathbb{R}) .
$$

But then, since $f(0), g(0), \kappa$, are different from zero, $g$ would change sign at $v=1 /(\kappa f(0))$, contrary to the supposition of Theorem 3. Thus (32) holds.

In the remaining case, $a \neq 0$, we define

$$
m(u)=1-a \ell(u) \quad\left(u \in \mathbb{R}_{+}\right) .
$$

Then (30) becomes

$$
m(u+v)=m(u) m(v) \quad\left(u \in \mathbb{R}_{+}, v \in \mathbb{R}_{+}\right) .
$$

By (29) and (37), $m(u)=0$ for all $u \in \mathbb{R}_{+}$would imply $h(v)=a$ and thus, by (28), $f(v)=0\left(v \in \mathbb{R}_{+}\right)$- but $f$ is nowhere 0 . So $m$ cannot be identically 0 . By (31), the values of $\ell$, just as those of $h$, are either all positive or all negative. If $a \ell(u)>0$, then $m(u)<1$ for all $u \in \mathbb{R}_{+}$; if $a \ell(u)<0$, then $m(u)>1$ for all $u \in \mathbb{R}_{+}$. Thus in both cases $m$ is bounded from one side by a positive constant. But then the solution of equation (38) can only be (cf. e.g. Aczél [1, pp. 38-39])

$$
m(u)=e^{\lambda u} \quad\left(u \in \mathbb{R}_{+}\right)
$$


for some nonzero constant $\lambda$. By (29), (37) and (39) we have (23)

$$
h(u)=\frac{a}{1-e^{\lambda u}} \quad\left(u \in \mathbb{R}_{+}\right) .
$$

Furthermore, from (28) and (26)), with the notation (remember, $f(0) \neq 0$ and $a \neq 0$ )

$$
\mu=\frac{a-f(0)}{f(0)}, \quad b=\frac{a g(0)}{f(0)},
$$

we have now the solutions (21) and (22) for $v \in \mathbb{R}_{+}$, that is,

$$
f(v)=\frac{a e^{\lambda v}}{\mu+e^{\lambda v}}, \quad g(v)=\frac{b}{\mu+e^{\lambda v}} \quad\left(v \in \mathbb{R}_{+}\right) .
$$

Again we show that the same holds for all $v \in \mathbb{R}$. Here too we take an arbitrary $v \in \mathbb{R}$ and a $u \in \mathbb{R}_{+}$such that $u+v \in \mathbb{R}_{+}$. Using (20), (23) and (40), we get

$$
f(v)=h(u)-\frac{h(u) g(v)}{g(u+v)}=\frac{a}{1-e^{\lambda u}}-\frac{a\left(\mu+e^{\lambda(u+v)}\right)}{b\left(1-e^{\lambda u}\right)} g(v) \quad(v \in \mathbb{R}) .
$$

Letting $u \rightarrow+\infty$, we obtain (remember, $\lambda \neq 0$ ) if $\lambda<0$,

$$
f(v)=a-\frac{a \mu}{b} g(v) \quad(v \in \mathbb{R}),
$$

and, if $\lambda>0$,

$$
f(v)=\frac{a}{b} \lim _{u \rightarrow+\infty} \frac{b-\mu g(v)}{1-e^{\lambda u}}-\frac{a}{b} \lim _{u \rightarrow+\infty} \frac{e^{\lambda v} g(v)}{e^{-\lambda u}-1}
$$

that is,

$$
f(v)=\frac{a}{b} e^{\lambda v} g(v) \quad(v \in \mathbb{R}) .
$$

If we put either (42) or (431) into (41), we get in both cases

$$
g(v)=\frac{b}{\mu+e^{\lambda v}} \quad \text { for all } v \in \mathbb{R},
$$

and, putting this too into (41), we get also

$$
f(v)=\frac{a e^{\lambda v}}{\mu+e^{\lambda v}} \quad \text { for all } v \in \mathbb{R} .
$$

So we have obtained (22) and (21) in full generality.

Finally we note that $f$ and $g$ are defined for all $v \in \mathbb{R}$, so the denominator $\left(\mu+e^{\lambda v}\right)$ in (21) and (22) should not be zero for any $v \in \mathbb{R}$, which is possible only if $\mu \geq 0$. We had already $a \neq 0, \lambda \neq 0$. Furthermore $g(0) \neq 0$, so $b=a g(0) / f(0) \neq 0$. Thus the constants are as stated in Theorem 3 .

Substitution shows that (21), (22) and (23) satisfy (20) with arbitrary $\mu \geq 0$, $a \neq 0, b \neq 0$ and $\lambda \neq 0$. This concludes the proof of Theorem 3 .

Remark. Actually, as pointed out also by the referee, the function $g$ in (36), $h$ in (33), and the function $f$ given by (35) satisfy (20) for $u \in R_{+}, v \in \mathbb{R}$ with $v, u+v \neq 1 /(f(0) \kappa)$; that is, these functions form a system of singular (conditional) solutions of equation (20). They do not satisfy, however, the conditions of Theorem 3 about $f$ and $g$ not changing signs; therefore they were eliminated in the proof. 


\section{Solution of the $F-G-\Phi$ equation}

We return now to equation (14) in Proposition 2, which is of the form (20) with

$$
f(v)=F^{\prime}(v), \quad g(v)=G^{\prime}(v) \quad(v \in \mathbb{R}), \quad h(u)=\Phi^{\prime}(u) \quad\left(u \in \mathbb{R}_{+}\right)
$$

and, by (11), (12) and (13), all conditions of Theorem 3 are satisfied. So

$$
F^{\prime}(v)=\frac{a e^{\lambda v}}{\mu+e^{\lambda v}}, \quad G^{\prime}(v)=\frac{b}{\mu+e^{\lambda v}} \quad(v \in \mathbb{R}), \quad \Phi^{\prime}(u)=\frac{a}{1-e^{\lambda u}} \quad\left(u \in \mathbb{R}_{+}\right),
$$

where $\mu \geq 0, \lambda \neq 0, a \neq 0, b \neq 0$ are constants. Moreover, by (12)

$$
b>0 .
$$

Integration in (44) yields $\left(-\frac{a}{\lambda} \ln \alpha\right.$ and $\frac{a}{\lambda} \ln \delta$ are the constants of integration)

$$
\begin{aligned}
& \Phi(u)=-\frac{a}{\lambda} \ln \left(\alpha\left|e^{-\lambda u}-1\right|\right) \quad\left(u \in \mathbb{R}_{+}\right), \\
& F(v)=\frac{a}{\lambda} \ln \left(\delta\left(\mu+e^{\lambda v}\right)\right) \quad(v \in \mathbb{R})
\end{aligned}
$$

(no absolute value signs are needed in (47) since $\mu+e^{\lambda v}>0$ ). If we had $\mu=0$, then (44) would give

$$
G^{\prime}(v)=b e^{-\lambda v}, \quad G(v)=-\frac{b}{\lambda} e^{-\lambda v}+C .
$$

Substitution into (9) with $v=0$ would yield

$$
\ln \left(\alpha\left|e^{-\lambda u}-1\right|\right)-\ln \delta=\ln \left(\alpha\left|e^{b\left(e^{-\lambda u}-1\right)}-1\right|\right),
$$

that is, with $x=e^{-\lambda u}-1(<0$ if $\lambda>0$ and $>0$ if $\lambda<0)$,

$$
|x|=\delta\left|e^{b x}-1\right|
$$

for all $x>0$ (if $\lambda<0$ ) or for all $x<0$ (if $\lambda>0$ ), which is nonsensical. Thus we have $\mu>0$ and, from (44),

$$
G(v)=-\frac{b}{\lambda \mu} \ln \left(\beta\left(1+\mu e^{-\lambda v}\right)\right) \quad(v \in \mathbb{R}) .
$$

(Here $-\frac{b}{\lambda} \ln \beta$ is the integration constant and again no absolute value sign is needed because $1+\mu e^{-\lambda v}>0$; of course $\alpha>0, \beta>0, \delta>0$.)

We substitute now (46), (47) and (48) into (92) and get, after multiplication by $-\frac{\lambda}{a}$ and taking exponentials

$$
\frac{\alpha\left|e^{-\lambda u}-1\right|}{\delta\left(\mu+e^{\lambda v}\right)}=\alpha\left|e^{-\lambda(G(u+v)-G(v))}-1\right|=\alpha\left|\left(\frac{e^{\lambda v}+\mu e^{-\lambda u}}{e^{\lambda v}+\mu}\right)^{b / \mu}-1\right| .
$$

Here let $e^{\lambda v} \rightarrow 0$ (i.e. $v \rightarrow+\infty$ if $\lambda<0$, while $v \rightarrow-\infty$ if $\lambda<0$ ):

$$
\frac{\left|e^{-\lambda u}-1\right|}{\delta \mu}=\left|\left(e^{-\lambda u}\right)^{b / \mu}-1\right| \text {. }
$$

This is possible only if $\delta=\frac{1}{\mu}$ and $b=\mu$ in which case (49) is indeed satisfied. Thus we have proved the following result. 
Theorem 4. Let $F: \mathbb{R} \rightarrow \mathbb{R}, \Phi: \mathbb{R}_{+} \rightarrow \mathbb{R}$ be strictly monotonic, $G: \mathbb{R} \rightarrow \mathbb{R}$ strictly increasing. They satisfy (9) if, and only if, there exist nonzero constants a, $\lambda$ and positive constants $\alpha, \beta, \mu$ such that

$$
\begin{aligned}
& F(v)=\frac{a}{\lambda} \ln \left(1+\frac{1}{\mu} e^{\lambda v}\right) \quad(v \in \mathbb{R}), \\
& G(v)=-\frac{1}{\lambda} \ln \left(\beta\left(1+\mu e^{-\lambda v}\right)\right) \quad(v \in \mathbb{R}),
\end{aligned}
$$

and

$$
\Phi(u)=-\frac{a}{\lambda} \ln \left(\alpha\left|e^{-\lambda u}-1\right|\right) \quad\left(u \in \mathbb{R}_{+}\right) .
$$

\section{Solution of the Marley-Luce problem}

We now turn our attention to the original problem, that is, to equation (5). In view of Proposition 1, and of (52), (51), (50),

$$
\begin{aligned}
\varphi(z) & =e^{\Phi(-\ln z)}=\left(\alpha\left|z^{\lambda}-1\right|\right)^{-a / \lambda}=A\left|z^{-k}-1\right|^{c} \quad(z \in] 0,1[), \\
\gamma^{-1}(y) & =\frac{1}{y} e^{-G(-\ln y)}=\left(\frac{\beta+\beta \mu y^{\lambda}}{y^{\lambda}}\right)^{1 / \lambda} \\
& =\left(\beta y^{k}+\beta \mu\right)^{-1 / k} \quad\left(y \in \mathbb{R}_{+}\right), \\
\psi\left(\gamma^{-1}(y)\right) & =e^{F(-\ln y)}=\left(1+\frac{1}{\mu} y^{-\lambda}\right)^{a / \lambda} \\
& =\left(1+\frac{1}{\mu} y^{k}\right)^{-c} \quad\left(y \in \mathbb{R}_{+}\right)
\end{aligned}
$$

(where $k=-\lambda \neq 0, c=-\frac{a}{\lambda} \neq 0, A=\alpha^{c}>0$ ). Since $\gamma$ is supposed to be decreasing and surjective, $\left(\lim _{y \rightarrow+\infty} \gamma^{-1}(y)=0\right.$ and) $\lim _{y \rightarrow 0} \gamma^{-1}(y)=1$. Thus, in (54), $k>0$ and $\beta=\frac{1}{\mu}$, so from (53), (54) and (55), with $B=\mu^{1 / k}>0$,

$$
\begin{aligned}
\varphi(z) & =A\left(z^{-k}-1\right)^{c} \quad(z \in] 0,1[), \\
\gamma(p) & =\left(\mu p^{-k}-\mu\right)^{1 / k}=\mu^{1 / k}\left(p^{-k}-1\right)^{1 / k} \\
& =B\left(p^{-k}-1\right)^{1 / k} \quad(p \in] 0,1[), \\
\psi(p) & =\left(1+\frac{1}{\mu} \gamma(p)^{k}\right)^{-c}=\left(1+\frac{1}{\mu} B^{k}\left(p^{-k}-1\right)\right)^{-c} \\
& =p^{k c} \quad(z \in] 0,1[) .
\end{aligned}
$$

Conversely, (5) is satisfied by (56), (57) and (58), whatever the constants $c \neq 0$, $k>0, A>0, B>0$. Also, $\varphi, \psi:] 0,1\left[\rightarrow \mathbb{R}_{+}\right.$are strictly monotonic and $\left.\gamma:\right] 0,1[\rightarrow$ $\mathbb{R}$ is strictly decreasing and surjective.

Thus we have the following answer to the Marley-Luce problem.

Theorem 5. The functions $\varphi, \psi:] 0,1\left[\rightarrow \mathbb{R}_{+}\right.$are strictly monotonic, $\left.\gamma:\right] 0,1[\rightarrow$ $\mathbb{R}_{+}$strictly decreasing, surjective and they satisfy $(5)$ if, and only if, there exist 
constants $A>0, B>0, k>0$ and $c \neq 0$ such that for all $z, p \in] 0,1[$

$$
\begin{aligned}
& \varphi(z)=A\left(z^{-k}-1\right)^{c}, \\
& \gamma(p)=B\left(p^{-k}-1\right)^{1 / k},
\end{aligned}
$$

and

$$
\psi(p)=p^{k c} .
$$

Corollaries. 1. If $\lim _{p \rightarrow 0}[p \gamma(p)]=1$, then $B=1$.

2. If $\lim _{z \rightarrow 1} \varphi(z)=+\infty$ is supposed, then $c<0$. Thus, $\varphi$ is strictly increasing and $\psi$ is strictly decreasing; furthermore the codomains of $\varphi$ and $\psi$ are $] 0,+\infty[$ and ] $1,+\infty[$, respectively.

This conforms with the application (cf. the Introduction and the beginning of Section 2).

\section{REFERENCES}

[1] J. Aczél, Lectures on Functional Equations and their Applications, Academic Press, New York/London, 1966. MR 34:8020

[2] E. Hewitt and K. Stromberg, Real and Abstract Analysis, Springer, New York/Heidelberg, 1975. MR 51:3363

[3] M. Kuczma, An Introduction to the Theory of Functional Equations and Inequalities, Państwowe Wydawnictwo Naukowe, Warszawa/Kraków/Katowice, 1985. MR 86i:39008

[4] A. A. J. Marley and R. D. Luce, A simple axiomatization of binary rank-dependent expected utility for gains (losses), submitted.

[5] F. Riesz and B. Szőkefalvi-Nagy, Functional Analysis, Dover, New York, 1990. MR 91g:00002

[6] A. W. Roberts and D. E. Varberg, Convex Functions, Academic Press, New York and London, 1973. MR 56:1201

Department of Pure Mathematics, University of Waterloo, Waterloo, Ontario, CANADA N2L 3G1

E-mail address: jdaczel@math.uwaterloo.ca

Current address: Institute for Mathematical Behavioral Sciences, SSP, University of California, Irvine, California 92697-5100

E-mail address: janos@aris.ss.uci.edu

Institute of Mathematics and Informatics, University of Debrecen, H-4010 Debrecen, Pf. 12, Hungary

E-mail address: maksa@math.klte.hu

Institute of Mathematics and Informatics, University of Debrecen, H-4010 Debrecen, PF. 12, Hungary

E-mail address: pales@math.klte.hu 